\title{
Developmental and reproductive patterns of Triatoma brasiliensis infected with Trypanosoma cruzi under laboratory conditions
}

\author{
Tiago G Oliveira', Filipe A Carvalho-Costa ${ }^{2}$, Taís F Gomes ${ }^{1}$, \\ Otília Sarquis', Ricardo Sposina ${ }^{1,3}$, Marli M Lima ${ }^{1 /+}$ \\ ${ }^{1}$ Laboratório de Eco-Epidemiologia da Doença de Chagas \\ ${ }^{2}$ Laboratório de Sistemática e Bioquímica, Instituto Oswaldo Cruz-Fiocruz, Av. Brasil 4365, 21045-900 Rio de Janeiro, RJ, Brasil \\ ${ }^{3}$ Laboratório de Tecnologia Enzimática, Instituto de Química, Universidade Federal do Rio de Janeiro, Rio de Janeiro, RJ, Brasil
}

The aim of this work was to study the interaction between Trypanosoma cruzi- 1 and Triatoma brasiliensis. A group of lst instar nymphs was initially fed on $\mathrm{T}$. cruzi-infected mice and a control group was fed on uninfected mice. From the second feeding onwards, both groups were otherwise fed on non-infected mice. The resulting adults were grouped in pairs: infected male/uninfected female, uninfected male/infected female, infected male and female and uninfected male/uninfected female. The infection affected only the 1st instar nymphs, which took significantly more time to reach the 2nd instar than uninfected nymphs. The differences in the molting time between the infected and uninfected nymphs from the 2 nd to the 5 th instars were not statistically significant. Both groups presented similar rates of nymphal mortality and reproductive performance was not significantly affected by infection in any of the treatments.

Key words: Triatoma brasiliensis - Trypanosoma cruzi - Chagas disease

The incidence rates of Chagas disease have been substantially reduced in Brazil and other South American countries following a successful effort to eradicate its main vector, Triatoma infestans (Coura \& Dias 2009). Nevertheless, Northeastern and Amazonian Brazil are considered regions that may present a special concern regarding control of the disease: human populations are in increasing contact with many autochthonous sylvatic triatomine species due to ecological disequilibrium and anthropic environmental transformations (Costa \& Lorenzo 2009, Fé et al. 2009, Guhl et al. 2009). Among the sylvatic species of triatomines inhabiting natural ecotopes in xerophytic ecosystems, Triatoma brasiliensis, Triatoma pseudomaculata and Rhodnius nasutus deserve special attention. These species, which have been found colonizing peridomestic ecotopes in Northeastern Brazil, are often infected with Trypanosoma cruzi (Sarquis et al. 2004, 2006, Lima \& Sarquis 2008). Given these observations, a better understanding of the biological aspects of these potential Chagas disease vectors in Northeastern Brazil is needed.

Although there is little evidence that $T$. cruzi infection can cause substantial harm to the invertebrate vector, the pathological interaction between $T$. cruzi and T. brasiliensis has not yet been studied. In the present study, we analyzed the effects of $T$. cruzi infection on the developmental and reproductive aspects of T. brasiliensis under laboratory conditions.

Financial support: FAPERJ, CNPq

+ Corresponding author: mmlima@ioc.fiocruz.br

Received 7 May 2010

Accepted 1 September 2010
The T. cruzi strain CE-4 (zymodeme 1) was isolated from a naturally infected $T$. brasiliensis specimen collected in Jaguaruana, state of Ceará, Northeastern Brazil (Pacheco et al. 2005). The strains were kept in cultures maintained in the diphasic medium, Neal Novel Nicolle/ Brain Heart Infusion, by serial passages for 21 days. On day 12 post-infection, Swiss-Webster mice were used as the source of infection. These mice had a mean parasitemia of $7.0 \pm 0.8 \times 10^{4}$ trypomastigotes per $\mathrm{mL}$. The mice were treated according to the procedure approved by the Animal Use and Care Committee at Oswaldo Cruz Foundation (license L-P0067-00).

First instar T. brasiliensis nymphs in the F1-generation bred in the laboratory were descendents from insects also collected in Jaguaruana. Ten days after eclosion from the eggs, 95 nymphs were fed on T. cruzi-infected mice and 95 nymphs were fed on uninfected mice. In both groups, the 1st instar nymphs ingested an average of $10 \pm 1.2 \mathrm{mg}$ of blood. From the second feeding, both groups were fed on non-infected mice every 20 days. The insects were weighed individually before and after each feeding. After the first feeding, nymphs from both groups were kept in individual glass vials $(19 \mathrm{~cm} \times 3 \mathrm{~cm})$ and maintained in climatic chambers with a controlled temperature of $29 \pm$ $1^{\circ} \mathrm{C}$ and $60 \pm 10 \%$ relative humidity.

Weekly observations, recording ecdyses and mortalities, were made. To avoid mortality during the earlier stages in the experimental group, only those insects from the 4th instar nymphs to the adult instars were checked for $T$. cruzi infection, which was done by compressing the abdomen gently and collecting the insect feces on slides. All of the insects in the infected group were checked to confirm infection. The gut content was diluted in phosphate buffered saline and examined for the presence of trypanosomatids by light microscopy at 400X.

The reproductive profile of the adult insects from both the infected and uninfected groups were studied using 
the following parameters: (i) pre-oviposition period (days), (ii) number of ovipositions (i.e., how often the females laid eggs, independently of the number of eggs laid), (iii) total number of eggs and (iv) number of fertile eggs (i.e., eggs that ecloded and produced nymphs). Four groups, each of them containing a mean of seven couples, were constituted: (i) infected male/uninfected female, (ii) uninfected male/infected female, (iii) infected male and female and (iv) uninfected male/uninfected female. The pairs were kept under the same laboratory conditions described above. The offspring of each cross were collected in separate vials. The completion of mating was verified by the presence of the spermatophore casing in the flasks.

The medians of numerical variables were compared with the Kruskal-Wallis test. Cumulative percentages were compared by the Kolmogorov-Smirnov test. The Mantel-Cox test was performed to compare the survival distributions of the distinct insect groups. Statistical significance was established as $p<0.05$. Analyses were performed using the software SPSS 15.0 (SPSS Inc, Chicago, Illinois, USA) and Bioestat 5.0 (Instituto de Desenvolvimento Sustentável Mamirauá, Tefé, Amazonas, Brazil).

In the group of uninfected 1st instar nymphs, $70 \%$ molted to the 2nd instar 25-30 days after eclosion, whereas in the infected 1st instar nymphs, 70\% molted to the 2 nd instar 45-50 days after eclosion. A comparison of the cumulative proportion of molting revealed a significant difference between the experimental and control groups $(\mathrm{p}<0.001$, Kolmogorov-Smirnov test). Although the infected group showed an interesting trend toward faster development, there was no significant difference between the two groups in the other nymphal stages (Fig. 1). The survival distributions for the adult insects were similar in both the infected and uninfected groups (data not shown).
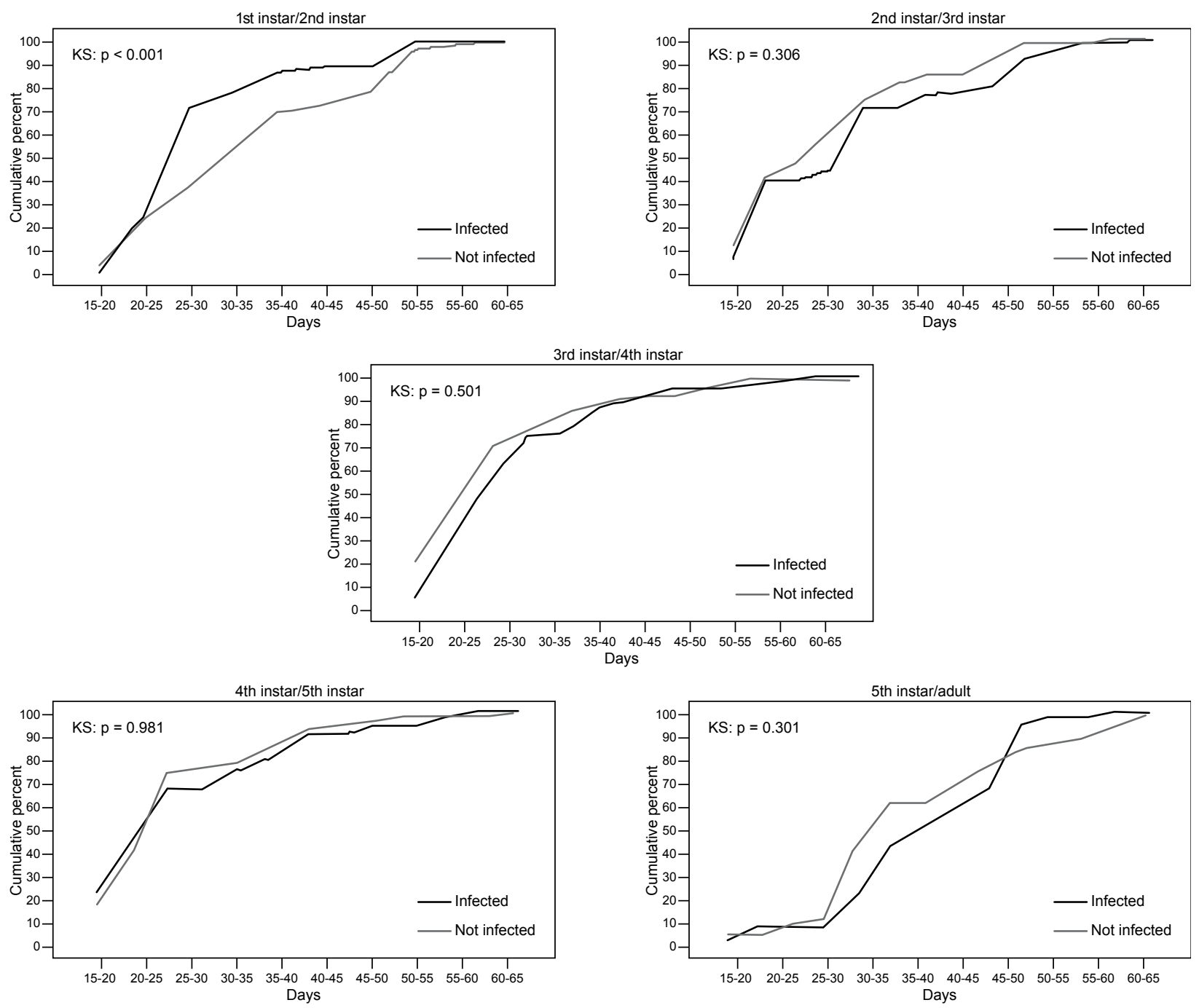

Fig. 1: cumulative percentages of molts plotted against age for Triatoma brasiliensis in uninfected and Trypanosoma cruzi-infected insects. KS: Kolmogorov-Smirnov test. 
A comparison of the data for number of ovipositions, pre-oviposition period in days, fecundity (total number of eggs) and fertility (number of fertile eggs) for the four distinct pairings did not reveal any significant differences in the medians of any of these reproductive parameters (Fig. 2).

This is the first study that aims to evaluate the influence of $T$. cruzi-infection on the developmental and reproductive parameters of T. brasiliensis. Uninfected 1st instar nymphs molted significantly earlier than infected ones, suggesting that $T$. cruzi infection could lead to a delay in the development of this nymphal stage. Interestingly, a trend toward faster development in the infected group, although interesting, was not statistically significant.

T. cruzi multiplies and differentiates in the digestive tract of triatomines. Between one and four weeks postinfection, the density of the parasites increases in the small intestine, after which the density increases more in the rectum (Schaub 1989b). The experimental infections performed in this study used a T.cruzi-1 strain that had been obtained from $T$. brasiliensis specimens captured in the same area as the specimens studied herein.

T. cruzi, unlike Trypanosoma rangeli, has been established to be harmful to mammals, mainly humans. In contrast, it seems to only be sub-pathogenic to the triatomines (Schaub 1988, 1989a, 1992, 1994), whereas T. rangeli is pathogenic to its triatomine vectors (Grewal 1957, Añez 1984, Añez et al. 1987). T. cruzi requires synergistic stressors, such as starvation or other envi- ronmental factors, to cause actual harm to the triatomines (Schaub 1992). Scanning electron microscopy demonstrated that the T. cruzi parasites create a carpet of 3-4 layers on the rectal cuticle of the insect, which could impair the uptake of nutrients (Schaub \& Böker 1987). In T. cruzi-infected Panstrongylus megistus, the reproductive parameters were significantly affected (Lima et al. 1992). T. cruzi-infected Rhodnius prolixus appear to have a shortened life span and may have a reduction in its egg-laying period (Neves \& Peres 1975). In addition, T. cruzi-infected nymphs needed up to 100 days to molt, whereas uninfected nymphs completed this developmental instar after 15-20 days (Reis dos Santos \& Lacombe 1985). Interestingly, these results are similar to our data on T. brasiliensis. Schaub (1988) argued that $T$. infestans development may be affected when the nymphs are housed separately. This isolation may have had some influence on our 1st instar nymphs, although the other nymphal stages, none of which showed any differences in development, were also kept isolated.

As strict blood feeders, Chagas disease vectors strongly depend on the possession of symbiotic bacterial flora in their guts (Eichler \& Schaub 2002); the deleterious effects of aposymbiosis in triatomines have been described. These bacteria - mainly actinomycetes - include species of Rhodococcus, Nocardia and Gordonia (Brecher \& Wigglesworth 1944). An experimental infection of the vector R. prolixus with $T$. rangeli, but not with T. cruzi or Blastocrithidia triatomae, reduced the
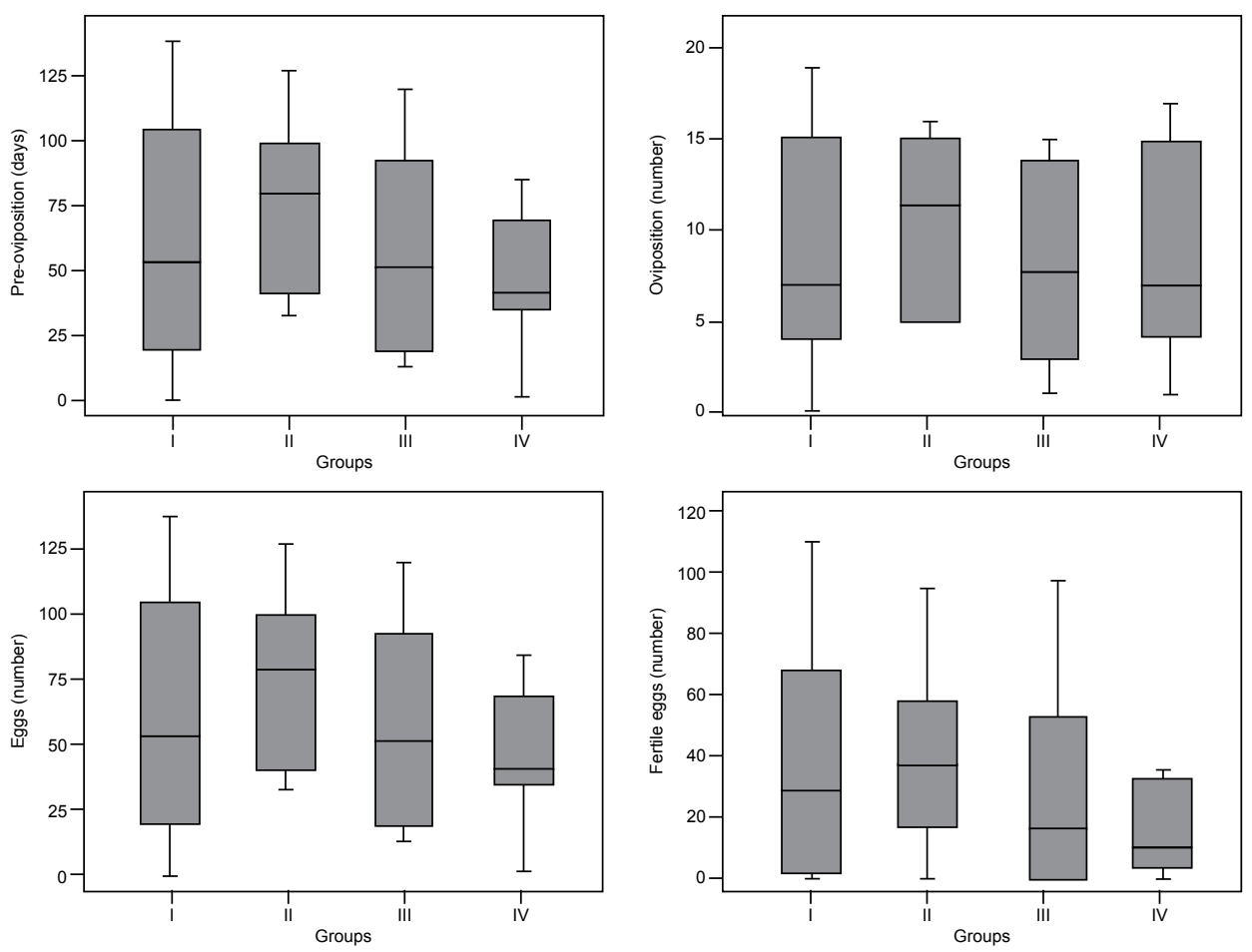

Fig. 2: box-plots displaying medians and interquartile ranges from pre-oviposition, oviposition, eggs and fertile eggs from Triatoma brasiliensis belonging to distinct couple groups. Differences were not statistically significant. I: infected male/uninfected female $(\mathrm{n}=6)$; II: uninfected male/infected female ( $n=9)$; III: infected male/female $(n=5)$; IV: uninfected male/female $(n=6)$. 
gut population of the symbiont Rhodococcus rhodnii. Infection of $T$. infestans with $B$. triatomae, but not with $T$. cruzi or $T$. rangeli, reduced the gut population of the symbiotic Nocardia spp. Given these observations, the pathogenic effects caused by trypasomatids could be a result of their interference with symbiotic bacterial populations (Eichler \& Schaub 2002). These studies, however, did not demonstrate a $T$. cruzi-mediated interference with the symbiotic flora of $R$. prolixus nor $T$. infestans.

In conclusion, our data suggest that $T$. cruzi infection does not significantly impair the reproduction parameters and mortality of $T$. brasiliensis, but does cause a developmental delay between the 1 st and 2 nd instar stages.

\section{REFERENCES}

Añez N 1984. Studies on Trypanosoma rangeli Tejera, 1920. VII Its effect on the survival of infected triatomine bugs. Mem Inst Oswaldo Cruz 79: 249-255.

Añez N, Nieves E, Cazorla D 1987. Studies on Trypanosoma rangeli Tejera, 1920. IX. Course of infection in different stages of Rhodnius prolixus. Mem Inst Oswaldo Cruz 82: 1-6.

Brecher G, Wigglesworth VB 1944. The transmission of Actinomyces rhodnii Erikson in Rhodnius prolixus Stal (Hemiptera) and its influence on the growth of the host. Parasitololy 35: 220-224.

Costa J, Lorenzo M 2009. Biology, diversity and strategies for the monitoring and control of triatomines - Chagas disease vectors. Mem Inst Oswaldo Cruz 104 (Suppl. I): 46-51.

Coura JR, Dias JCP 2009. Epidemiology, control and surveillance of Chagas disease - 100 years after its discovery. Mem Inst Oswaldo Cruz 104 (Suppl. I): 31-40.

Eichler S, Schaub GA 2002. Development of symbionts in triatomine bugs and the effects of infections with trypanosomatids. Exp Parasitol 100: 17-27.

Fé NF, França MS, Carvalho-Costa FA 2009. Reassessing the entomological investigation around the first autochthonous case of Chagas disease in Western Brazilian Amazon. Mem Inst Oswaldo Cruz 104: 121-123.

Grewal MS 1957. Pathogenicity of Trypanosoma rangeli Tejera, 1920 in the invertebrate host. Exp Parasitol 6: 123-130.

Guhl F, Pinto N, Aguilera G 2009. Sylvatic triatominae: a new challenge in vector control transmission. Mem Inst Oswaldo Cruz 104 (Suppl. I): 71-75.
Lima MM, Pereira JB, dos Santos JAA, Pinto ZT, Braga MV 1992. Development and reproduction of Panstrongylus megistus (Hemiptera: Reduviidae) infected with Trypanosoma cruzi, under laboratory conditions. Ann Entomol Soc Am 85: 458-461.

Lima MM, Sarquis O 2008. Is Rhodnius nasutus (Hemiptera: Reduviidae) changing its habitat as a consequence of human activity? Parasitol Res 102: 797-800.

Neves DP, Peres RB 1975. Aspectos da biologia do Rhodnius prolixus quando alimentado em animais sadios ou infectados com o Trypanosoma cruzi. Rev Bras Biol 35: 317-320.

Pacheco RS, de Brito CM, Sarquis O, Pires MQ, Borges-Pereira J, Lima MM 2005. Genetic heterogeneity in Trypanosoma cruzi strains from naturally infected triatomine vectors in Northeastern Brazil: epidemiological implications. Biochem Genet 43: 519-530.

Reis dos Santos J, Lacombe D 1985. Estudos relativos à duração da ecdise e ovoposição de Triatoma infestans infectado pelo Trypanosoma cruzi. An Acad Bras Cienc 57: 127.

Sarquis O, Borges-Pereira J, Mac Cord JR, Gomes TF, Cabello PH, Pereira JB, Lima MM 2004. Epidemiology of Chagas disease in Jaguaruana, Ceará, Brazil. I. Presence of triatomines and index of Trypanosoma cruzi infection in four localities of a rural area. Mem Inst Oswaldo Cruz 99: 263-270.

Sarquis O, Sposina R, de Oliveira TG, Mac Cord JR, Cabello PH, Borges-Pereira J, Lima MM 2006. Aspects of peridomiciliary ecotopes in rural areas of Northeastern Brazil associated to triatomine (Hemiptera, Reduviidae) infestation, vectors of Chagas disease. Mem Inst Oswaldo Cruz 101: 143-147.

Schaub GA 1988. Development of isolated and group-reared first instars of Triatoma infestans infected with Trypanosoma cruzi. Parasitol Res 74: 593-594.

Schaub GA 1989a. Does Trypanosoma cruzi stress its vectors? Parasitol Today 5: 185-188.

Schaub GA 1989b. Trypanosoma cruzi: quantitative studies of development of two strains in small intestine and rectum of the vector Triatoma infestans. Exp Parasitol 68: 260-273.

Schaub GA 1992. The effects of trypanosomatids on insects. $A d v$ Parasitol 31: 255-319.

Schaub GA 1994. Pathogenicity of trypanosomatids on insects. Parasitol Today 10: 463-468.

Schaub GA, Böker CA 1987. Colonization of the rectum of Triatoma infestans by Trypanosoma cruzi studied by scanning electron microscopy: influence of blood uptake by the bug. Parasitol Res 73: 417-420. 